\section{Narkose schadet Baby-Hirn}

Eine Vollnarkose bei Kleinkindern im Alter von weniger als einem Jahr kann spätere Phasen der Entwicklung negativ beeinflussen, so eine amerikanischen Studie. Zu diesem Schluss kamen die Anästhesisten um Prof. Robert Block von der Universität in lowa City, indem sie bei 287 Kindern, die als Babys eine Vollnarkose erhalten hatten, die Ergebnisse des lowaTests auswerteten. Bei diesem Test, mit dem allgemeine intellektuelle und kognitive Leistungen geprüft werden, erhielten die Probanden, im Vergleich zur Allgemeinbevölkerung, im Schnitt einen deutlich niedrigeren Wert (43 vs. 50 Punkte). Als ausschlaggebend erwies sich außerdem die Dauer der Anästhesie.

Block Rl et al, Anesthesiology 2012 (online first)

\section{Wunde? Hautzellen aufsprühen!}

Regeneration der Hautzellen und somit eine schnellere Wundheilung chronischer Beingeschwüre, das verspricht nun ein Spray. Eingebettet in einer Fibrinmatrix enthält das neue Heilmittel aus der Dose Keratinozyten und Fibroblasten, die die Regenerationsprozesse der eigenen Zellen anregen sollen. Dass es funktioniert, belegt eine randomisierte placebokontrollierte Phase-II-Studie an 228 Patienten. Als optimale Dosis erwies sich eine Zellkonzentration von $0,5 \times 106 / \mathrm{ml}$ alle 14 Tage. Dabei betrug die Heilungsrate $70 \%$ gegenüber $46 \%$ mit der Kontrollbehandlung.

Kirsner RS et al, Lancet 2012 (online first)

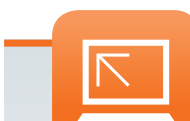

springermedizin.de aufTwitter

Auch im Herbst heißt es:

„Lauschen" Sie unserem

Zwitschern und werden Sie

zum „Follower"!

- twitter.com/springermedizin

Auch das noch!

\title{
Bierbauch fördert Blasenschwäche
}

Männer mit zentraler Adipositas, vulgo Bierbauch, riskieren nicht nur kardiovaskuläre Erkrankungen und Erektionsstörungen, sondern auch Miktionsprobleme, legt eine Studie der Cornell University in New York nahe.

Für die Studie waren die Daten von 409 konsekutiven LUTS-Patienten im Alter von 40 bis 91 Jahren (im Median 58 Jahre) ausgewertet worden. Von ihnen hatten $37,5 \%$ einen Taillenumfang unter $90 \mathrm{~cm}$, $33,5 \%$ zwischen 90 und $99 \mathrm{~cm}$ und $29 \%$ von $100 \mathrm{~cm}$ und mehr.

Mit steigendem Taillenumfang erhöhte sich die Miktionsfrequenz: 16\%, 27\% und $39 \%$ der Männer mussten innerhalb von 24 Stunden öfter als achtmal Wasser lassen. Gleichzeitig nahm der Anteil der
Männer mit Nykturie (mindestens zwei nächtliche Toilettengänge) von 15\% über $29 \%$ auf $44 \%$ zu. Eine schwere LUT-Symptomatik (International Prostata Symptom Score, IPSS 20-35) war zumindest bei den Männern mit mehr als $100 \mathrm{~cm}$ Taillenweite signifikant häufiger.

Der PSA-Spiegel korrelierte ebenfalls mit dem Taillenmaß, mit Werten von minimal 1,87 bis maximal 3,96 ng/dl. Erwartungsgemäß stieg mit dem Taillenumfang auch das Risiko für eine erektile Dysfunktion sowie für Bluthochdruck, Diabetes und KHK.

Den Miktionsprobleme könnte ein durch den erhöhten Insulinspiegel gefördertes Prostatawachstum zugrunde liegen, so die Forscher.

(bs)

\section{Trotz Nikotinabstinenz}

\section{Lungenkrebs liegt auch in der Familie}

Nie geraucht - und trotzdem Lungenkrebs! Die umfangreichste internationale Untersuchung mit fast 50.000 Teilnehmern legt nahe, dass das Lungenkrebsrisiko trotz lebenslanger $\mathrm{Ni}$ kotinabstinenz um fast das Zweifache erhöht ist, wenn Geschwister an Lungenkrebs erkrankt sind.

Im Rahmen der Erforschung genetischer Faktoren beim Lungenkrebs hat eine internationale Forschergruppe um Dr. Michele Coté aus Detroit die Auswertung der gepoolten Daten von insgesamt fast 50.000 Teilnehmern vorgelegt. Knapp 24.400 Teilnehmer hatten Lungenkrebs.

Das Ergebnis: Menschen mit einem an Lungenkrebs erkrankten Angehörigen der ersten Generation hatten generell ein um das 1,51-Fache erhöhtes Risiko, selbst daran zu erkranken. Die adjustierten ORWerte betrugen in der Studie 1,25, wenn der Vater erkrankt war, 1,37, wenn die Mutter erkrankt war und 1,82 , wenn ein Geschwister ein Bronchialkarzinom hatte.

Rauchen erhöht offensichtlich zusätzlich das Erkrankungsrisiko. Menschen, die erkrankte Angehörige der ersten Generation haben und irgendwann in ihrem Leben geraucht haben oder rauchen, weisen nämlich ein um fast das 3,2-Fache erhöhtes Risiko für Lungenkrebs auf.

Menschen mit einem solchen erhöhten Risiko für diese Tumorart sollten dementsprechend bei einem Lungenkrebs-Screening teilnehmen, so die Autoren.

(ple)

Coté ML et al, Eur J Cancer 2012 48/13:1957 